\title{
Una mirada al teatro profesional en la Audiencia de Charcas a finales del siglo XVI: el caso del autor de comedias Francisco de Morales
}

\author{
A look at professional theatre \\ at the end of the 16th century in the Audiencia de Charcas: \\ the case of the comedian Francisco de Morales. \\ Laura Paz Rescala \\ Università Ca' Foscari Venezia-Italia \\ Universidad de Sevilla-España \\ lauraagar.pazrescala@unive.it
}

\section{Resumen}

En este artículo me propongo reconstruir — principalmente a través de documentación notarial de La Plata-el paso por Charcas, en la década de 1590, de Francisco de Morales, autor de comedias y para entonces ya famoso en Lima. En el marco de este análisis, presento algunas conclusiones sobre el surgimiento del teatro profesional en el territorio, las cuales me conducen a mostrar cómo Charcas, desde muy temprano, ha sido esencial para la conformación de circuitos de teatro profesional en el Virreinato del Perú.

Palabras clave: Charcas, compañías teatrales, teatro profesional.

\begin{abstract}
In this article I intend to reconstruct - mainly through notarial records of La Plata - the presence in Charcas, during the 1590s, of Francisco de Morales, an autor de comedias by the time already famous in Lima. Within this framework I propose some conclusions about the emergence of professional theater in the territory that allow me to show how Charcas, since a very early period, was essential to the conformation of professional theatre circuits in the Viceroyalty of Perú.
\end{abstract}

Key words: Charcas, theatre companies, professional theatre.

Recibido: 19 de mayo de 2020 • Aceptado: 31 de julio de 2020

\author{
Autoctonía. Revista de Ciencias Sociales e Historia Vol. IV, Nº 2, Julio-Diciembre 2020, 257-268 \\ ISSN 0719-8213 \\ DOI: http://dx.doi.org/10.23854/autoc.v4i2.102
}

(ㄷ) (1) (2)(2) 
La necesidad de comprender la manera en que se puede afrontar un tema tan complejo como el desarrollo del teatro en Charcas durante el periodo colonial, ha conducido a que distintos estudiosos elaborasen lo que podríamos llamar estados de la cuestión. Estos no son sino intentos de demarcar un territorio historiográfico para invocar nuevos estudios y hacerles, en lo posible, de guía. Podría considerarse el primero de aquellos el capítulo sobre teatro que propone Teresa Gisbert en su Esquema de la literatura virreinal en Bolivia (1968). Resulta interesante la relevancia que se da, dentro de dicho esquema, a la documentación que Marie Helmer (1960), pocos años antes, encontró en los fondos de escrituras notariales potosinas. Interesante porque el estudio de Helmer, centrado en la actividad en la Villa Imperial a principios del siglo XVII del afamado autor de comedias Gabriel del Río, es el único, hasta el día de hoy, que se dedica exclusivamente al teatro profesional en la zona charqueña.

En el 2000, Ana Forenza y Josep M. Barnadas proponen sus "Noticias sobre el teatro en Charcas (siglos XVI-XIX)”, donde, además de prestar particular atención a las narraciones de fastos ofrecidas por el cronista Arzáns de Orsúa y Vela, dan cuenta de nuevos documentos sobre representaciones teatrales hallados por ellos mismos en los fondos del ABNB. El 2006 y el 2008 Andrés Eichmann publica, respectivamente, sus Notas sobre el teatro en Charcas y sus Nuevas notas sobre el teatro en Charcas, artículos en los que, además de ir actualizando el estado de la cuestión, incluye un resumen de algunos de sus más importantes aportes a este tema: 1 . la construcción y el estudio de un corpus de música incidental (es decir, piezas musicales insertas en medio de una puesta en escena teatral) conservado en el ABNB y en el ABAS; 2. el hallazgo, estudio y edición crítica de un conjunto de diez y ocho obras teatrales breves, en ningún caso anteriores a finales del sigloXVII, en el Convento de Santa Teresa de Potosí, entre cuyas paredes habrían tenido lugar las representaciones (Eichmann y Arellano, 2005).

Al analizar los trabajos referidos, salta a la vista un problema: la investigación acerca del teatro en Charcas está en un estado que podríamos calificar como disperso. Motivo por el cual, continuamente, ante los nuevos descubrimientos, los historiadores tienen la necesidad de hacer estados de la cuestión que ayuden a ubicarlos. Si bien hay estudios, de mucho valor, sobre ciertas obras o ciclos festivos en específico, creo que todavía no sabemos cómo tender el hilo entre ellos ${ }^{1}$. No sabemos por dónde comenzar a escribir la historia del teatro charqueño. Sucede que el teatro es un campo de investigación muy vasto que, además, no puede comprenderse en su plenitud si no se estudia en el marco de la historia de la fiesta y el espectáculo. En esta línea, creo que resulta útil el concepto de práctica escénica de Joan Oleza.

La práctica escénica es una práctica social compleja y, como tal, nace de condicionamientos y espacios ideológicos y produce efectos ideológicos, y en su despliegue integra y orienta toda una serie heterogénea de actos sociales (textos, representaciones, hechos legislativos, compañías, público, preceptivas...) que, si bien generan ideología específica por sí mismos, se integran en un gesto ideológico global, el de la práctica como 
tal, resultante de las relaciones de fuerza entre estos actos (1984, 9-10).

En Charcas podríamos diferenciar tres tipos de prácticas escénicas. En primer lugar, la misional, documentada en el territorio, por ahora, solo como estrategia evangelizadora de la orden jesuítica. En segundo lugar, la escolar: teatro didáctico, de cuna humanista, ejecutado principalmente al interior de las escuelas. Y, en tercer lugar, la práctica profesional, el teatro de los comediantes de oficio (el cual, claro, hace de modelo para montajes teatrales realizados por aficionados en los más variados espacios, como conventos o palacios). Estas categorías se van reformulando y van dialogando de distintas formas a lo largo del tiempo, pero cada una requiere una metodología de investigación propia y solo teniendo esto en cuenta se puede plantear una historia al respecto.

Siguiendo esta estela, en este artículo me concentraré en la que he designado como "práctica escénica profesional" y ofreceré una primera aproximación a la llegada a la Audiencia de comediantes de oficio, a finales del siglo XVI. Este trabajo se enmarca en una investigación de más largo aliento que se propone, en un futuro, llegar a reconstruir el proceso de profesionalización teatral en el territorio. Por supuesto, hay que lidiar con la devastadora pérdida de registros. Dos verdaderas catástrofes afectan directamente esta labor de investigación: en primer lugar, la pérdida de las actas del Cabildo secular de La Plata para el periodo que nos concierne, donde, de seguro, se encontraban los datos relativos a los distintos espectáculos públicos que gestionaba esta institución. Y, en segundo lugar, la desaparición de gran parte de los legajos de escrituras públicas de Potosí, donde habríamos podido encontrar más datos sobre diversas transacciones económicas realizadas por los autores de comedias que pasaron por la Villa Imperial en sus momentos de más esplendor. De hecho, el grueso de la aun limitada documentación sobre la que se puede comenzar a escribir esta historia - al menos para su periodo inicial- se encuentra en registros notariales de La Plata y en las actas que se han conservado del Cabildo secular potosino. Sumado esto, claro, a la información que nos llega más bien a través de documentos emitidos en Lima, pues la profesionalización teatral nació de la mano de la conformación de circuitos de representación que abarcaban distintas zonas del Virreinato.

Ahora bien, al decir proceso de profesionalización me refiero a aquel momento en el cual el teatro se convierte en un oficio que permite a sus miembros vivir, aunque sea parcialmente, de su ejercicio. Como ya tantos estudios han mostrado, este proceso en España se inició hacia la década de 1540. Los oficios y gremios fueron esenciales, pues para entonces tenían mucha experiencia en la organización de espectáculos, teatrales y parateatrales, destinados al lucimiento de las celebraciones públicas realizadas en los centros urbanos. De sus bases surgieron las primeras personas que decidieron hacer del teatro un oficio más, que debía ser pagado. Es necesario tener en cuenta las condiciones sociales que se requirieron para que esto sucediera. Fue importante que las ciudades tuvieran una economía consolidada, pues, al menos en el ámbito hispano, no se conoce ejemplo de profesionalización teatral que no haya tenido como punto de apoyo la disponibilidad, por parte 
del Ayuntamiento, de pagar a representantes para que se ocuparan del entretenimiento del Corpus Christi. Festividad por demás significativa en una España desbordante de símbolos de poder y profundamente contrarreformista ${ }^{2}$. Por otro lado -y lo digo de la mano de José Luis Canet (1997) un pilar para este proceso fue la conformación de lo que podríamos llamar una seudoburguesía: un sector de población que gozara de algo de tiempoy de dinero excedente, disponible para actividades de ocio, como ir al teatro. Las distintas ciudades americanas, poco a poco, cada una a su ritmo, fueron alcanzando estas condiciones. Así lo hicieron, tanto La Plata como Potosí, durante las últimas décadas del siglo XVI.

La primera noticia documentada que se tiene de un pago para la representación de comedias en el Corpus Christi de la Villa Imperial de Potosí (y de todo el territorio charqueño) se encuentra en el acta de Cabildo secular correspondiente a la sesión del 3 de mayo de $1593^{3}$ :

En este cabildo se trató como ya estaba de próximo la fiesta de Corpus Cristiy que, para solenizalla según tan gran fiesta requería, convenía nombrar personas que la tomasen a cargo, así en entapizar las calles, regallas y por ellas barrer y enramallas, como en sacar danzas, hacer dos comedias, una el propio día de corpus como otro el octavario. Y que para esto se hablen a las personas que están en esta villa que representan para que lo hagan. Para lo cual se dio comisión en forma a los dichos fieles ejecutores y poder para que como es uso y costumbre lo hagan de manera que no falte cosa alguna ${ }^{4}$.
Este documento fue citado por Joseph Barnadas y Ana Forenza (2000), quienes, sin embargo, leen mal la fecha y adelantan una década, a 1583, la noticia. En sesiones capitulares anteriores a 1593 - estudiadas, en este sentido, por Pablo Quisbert (2008) - la ciudad ya se había mostrado dispuesta a invertir en el lucimiento de esta festividad. Por ejemplo, en 1587 se explicita que los fieles ejecutores deben ocuparse de que los oficiosy "otras personas" salgan a la procesión con sus "cofradías e ceras e danzas y otro regocijos" y de que se aderecen las calles para la ocasión ${ }^{5}$. Parecería que, para este año, todavía el grueso de los espectáculos que podríamos considerar parateatrales - danzas e invenciones - corrían a cargo de los oficios de la ciudad. Sin embargo, en 1593, como demuestra el documento citado, el mismo Cabildo se dispuso a pagar por dos comedias distintas siguiendo el modelo estable, para entonces, en la ciudad de Los Reyes ${ }^{6}$.

Parto de esta noticia para dar pie al tema más específico de este artículo: la presencia en Charcas del autor de comedias Francisco de Morales, documentada, como mostraré, a partir de 1593. ¿Quién sabe si fue la presencia en la zona de un autor de comedias que ya tenía experiencia en la organización de los espectáculos para el Corpus Christi la quellevó al Cabildo a pagar por comedias? Estas son conjeturas, pero creo que la coincidencia de fechas debe ser señalada.

Tenemos noticias de la actividad en Los Reyes de Francisco de Morales desde 1582, año en el cual llega con mucha fuerza a los escenarios limeños para constituirse en su indiscutible protagonista. Su historia no es sino el mejor testimonio que ha 
llegado hasta nuestros días de lo que fue el proceso de profesionalización teatral en el Virreinato del Perú durante las últimas décadas del siglo XVI. Mis estudios sobre su carrera en Lima - que ahora sintetizo apresuradamente-me llevan a ver en él un verdadero hombre de teatro que, llegado de España, habitó en el virreinato un desfase. Desde la década de 1580, intentó lo que nadie en el Perú antes que él: vivir de hacer teatro. No siempre lo logró. Debió acudir de cuando en cuando a tratos comerciales de otra naturaleza o endeudarse hasta quedar tras las rejas. Pero sus intentos no fueron poco fructíferos. Llevan, sin ir muy lejos, durante la segunda mitad de la década de 1590, a la creación del corral de comedias de Santo Domingo en Lima que, según mis cálculos, es uno de los motivos por los que hacia 1598 comenzaron a firmarse contratos para la conformación de compañías teatrales en la capital virreinal. Morales, que es quien impulsa la creación de este primer teatro oficial en Lima, no logra ejercer su oficio en dichos predios; para entonces, además de estar transcurriendo sus últimos años de vida, tuvo, como veremos, varios problemas legales que no le permitieron siquiera permanecer en la capital.

Nuestro comediante, naturalmente, para forjar una carrera teatral, no podía someterse a los dictámenes del calendario festivo y a la variante disponibilidad del Cabildo de pagar por representaciones. Podemos ver, por una escritura notarial de 1584, que ya este año alquiló en Lima el corral de una casa de vivienda para convertirlo en corral de representaciones ${ }^{7}$. Al menos, desde 1586, comenzó a moverse por distintos lugares del Perú, lo que muestra que vio también en la itinerancia una manera de sostener su profesión. Sin ir muy lejos, como ya nota Lohmann Villena (1945: 61), el Cabildo, en su sesión del 2 de mayo, ordenó explícitamente que se escribiera al corregidor del partido de Ica para ordenar de mandar a Francisco de Morales a Lima, para que se encargue de las representaciones del Corpus Christi ${ }^{8}$. Sabemos que para 1590 se encontraba en Lima, y que tenía mucha fama, pues se le encargó la representación de una comedia para el suntuoso recibimiento que se realizó en la ciudad al virrey García Hurtado de Mendoza ${ }^{9}$.

En 1593 lo encontramos, junto a su esposa, María Rodríguez, como residente en la ciudad de La Plata (Sucre). Pudieron haber elegido asentarse en la capital charqueña por muchas razones, como por ejemplo la cercanía con Potosí. Ya he traído a colación la hipótesis de que Morales pudo haberse encargado del Corpus Christi potosino de este año. Ahora, veamos algunos de los datos documentados. Morales y su esposa, para diciembre de 1593, parecen tener todas las intenciones de permanecer en La Plata. El 7 de diciembre, un indio llamado Juan Yucra se asienta a trabajar con él-_quien en la escritura viene explícitamente señalado como "autor de comedias"- por un $a \tilde{n} 0^{10}$. No se especifican las labores que el siervo tendría que cumplir.

El 23 de diciembre, Morales firmó una escritura sin duda importante. Él, junto con su esposa, fueron obligados a pagar a Lucas de Alarcón 2.644 pesos de plata ensayada: el resto de una deuda de 5.500 pesos. De esta deuda, en el momento de la escritura, lograron pagar 1.000 y se comprometieron a pagar los 1.644 restantes antes del día de San Juan de 1594. Estamos ante la principal 
adquisición que hicieron los esposos en La Plata: lo que compran es "un mesón y una tienda de pulpería que es en la calle que va de la iglesia de San Agustín a la Villa de Potosí"11. En el mismo documento se obliga a no hacer modificaciones al local hasta haber cumplido con todo el pago. Poco después, el 29 de diciembre, a Morales se le obliga a pagar al mercader Bernardino Jiménez 1.362 pesos corrientes por una serie de suministros: diez arrobas de azúcar, sesenta piezas de ropa de abasca ${ }^{12}$ para hombres y mujeres, doce caparazones ${ }^{13}$ y 200 varas de sayales ${ }^{14}$. El pago lo tendría que realizar en un plazo máximo de cuatro meses. ¿Para qué tantos géneros y ropajes? La respuesta más evidente parecería ser que Morales, por ahora, se iba a disponer a comerciar en su nueva pulpería.

En los primeros tres meses del año siguiente, encontramos al autor de comedias, que en la documentación aparece como tal, comprando más géneros. Cabe apuntar que -si bien personas de todos los oficios en muchas ocasiones se daban al comercio de este tipo de mercancía- el oficio teatral estaba particularmente ligado, a través del vestuario o 'hato' que llevaban las compañías para sus actores, al negocio con géneros y ropajes (García García, 1996). El tres de enero, por ejemplo, a Morales se le obliga, con Andrés Martínez de Guilléstegui, a pagar 935 pesos de plata corriente, con un plazo de pago hasta el día de San Juan, por la compra de ochenta y cinco piezas de ropa ${ }^{15}$. El 11 de marzo, por el mismo tipo de mercadería, se endeudó con Alonso Granero por 650 pesos de plata corriente ${ }^{16}$. Entre enero y febrero de este año, además, realiza algunas transacciones de compra y venta de esclavos ${ }^{17}$.
Pese a lo dicho, algo tuvo que suceder a inicios del año de 1594 para que Morales desistiera de su decisión de establecerse en La Plata y regresara a Lima. Donde, ya en octubre, lo encontramos firmando el arrendamiento del espacio del convento de Santo Domingo que convertirá en corral de comedias $^{18}$. Una posibilidad es que se hubiera trasladado porque no lograba terminar de saldar allí la gran cantidad de deudas que había contraído entre finales de 1593 y principios de 1594. Sabemos, con certeza, que no logró pagar todas, pues el 13 de febrero de 1596 el mercader Bernardo Jiménez, con quien vimos que nuestro autor se endeudó en diciembre de 1593, dio poder a Pedro Guillén Mexía para que cobrara de Morales, que ya estaba en Lima, "mil trescientos sesenta y tantos pesos de plata" 19 ; es decir, el mismo monto de la deuda que contrajera tres años atrás y dejara sin saldar en La Plata.

Fuera como fuese, al regresar a Lima, las deudas continuaron persiguiéndolo y lo llevaron tras las rejas. Se endeudaba constantemente, pero continuaba invirtiendo. Estaba, claramente, intentando construir algo. En La Plata, intentó hacer funcionar una pulpería, que quién sabe si planeaba convertir en teatro, pues en el mismo documento se afirma que podría hacer remodelaciones cuando terminara de pagar su deuda. No funcionó. En Lima intentó fundar el primer corral de comedias y lo logró. Aunque él no pudo gozar de sus beneficios.

Sabemos de sus periodos en prisión por una serie de fianzas otorgadas en Lima, que permiten que sea liberado por periodos determinados. Ahora no me detendré en todas las que he visto, sino 
solamente en la que parece tener más relevancia. El 25 de septiembre de 1596, Miguel Sánchez de la Parra y Francisco Hernández Mudara se constituyen en fiadores de Francisco de Morales como respuesta a una carta que habría sido despachada por el juez de provincia de La Plata, debido a una deuda del autor de comedias por la cuantía de 999 pesos de a nueve reales el peso. Así las cosas, los fiadores se comprometen a que en el plazo de seis meses Morales se presentará en la cárcel de la Villa Imperial de Potosín ${ }^{20}$. Y esta es una de las últimas noticias que atestiguan la presencia de Morales en Los Reyes. Solamente trece días antes de la firma de la fianza citada, el 12 de septiembre de 1596, Morales cede el corral de Santo Domingo a Simón Fernández Casteloblanco ${ }^{21}$. De seguro la carta que envía el juez de provincia de La Plata ya había llegado a Lima. Es difícil no ver una relación entre estos dos hechos. El 2 de abril de 1599 Francisco de Morales se encuentra en La Plata. Presenta una escritura por la cual se aparta de los derechos que tuviera sobre el mesón y la pulpería y los traspasa a dos nuevos propietarios ${ }^{22}$. Luego, regresa a Los Reyes, donde, según refiere Lohmann Villena, muere el 21 de mayo de 1600 (1945: 55).

Es imposible que Francisco de Morales, durantesu estadía en Charcas, no se haya dedicado también al teatro, para empezar, por el mismo hecho que en los documentos notariales aquí producidos se lo conoce, específicamente, a través de su oficio de comediante. Es cierto que no logró vivir solo de este oficio, que como oficial cómico no logró el éxito esperado, pero fue el protagonista del camino que condujo a que pronto otros lo hicieran. $\mathrm{Su}$ vida profesional no transcurre en un mismo lugar, se mueve desde Lima, Ica, Cusco, hasta La
Plata y Potosí. El teatro profesional es como el comercio: implica romper fronteras y Morales fue uno de los primerísimos que, en el Perú, lo supo. Las ciudades que entonces tenían alguna de las cualidades que antes señalé como necesarias para la profesionalización, fueron colaborando para que este proceso se cristalizara; entre ellas, destacan La Plata y Potosí. Esta última, donde desde bastante antes las élites locales se empeñaban en poner en pie las fiestas más estupendas. Ciudad que era, además, gran imán de comerciantes; y una característica de las primeras compañías oficiales de teatro del Perú fue justamente que, cuando se presentaba la posibilidad, comerciaban. Este era uno de alicientes, como resulta claro al revisar la documentación notarial conservada en Lima, que conducía a las compañías teatrales, desde inicios del siglo XVII, a la Villa Imperial (la que, de hecho, se convirtió en el segundo eje, después de la capital virreinal, para las giras teatrales).

Un caso que me interesa destacar para demostrar que es un mismo movimiento el que impulsa, desde finales del siglo XVI, la presencia de profesionales del teatro en la Audiencia de Charcas y en la de Lima, es el caso de la compañía de comedias de Francisco Pérez de Robles y Miguel de Burgos, concertada en el Callao el 28 de junio de $1599^{23}$. Esta compañía es muy famosa porque se suele conocer - de manera a mi parecer muy simplista - como la primera compañía del Virreinato del Perú (Rosenbach, 1939; Finque, 2019). Se tendió a considerar que los miembros de la compañía llegaron de España y que, en su paso hacia la capital, primero se detienen en el Callao. Nada más equivocado. He encontrado un arrendamiento realizado por Pérez de Robles de 
un patio en la calle de San Agustín en el cercado de Lima (donde luego se edificará efectivamente un teatro) en marzo de ese mismo año, es decir, más o menos tres meses antes del contrato en el Callao ${ }^{24} ; \mathrm{y}$, más importante para lo que ahora nos interesa, Pérez de Robles y Miguel de Burgos no llegaron a Lima en 1599 desde España, lo hacen desde Charcas.

En efecto, entre los años 1597 y 1598 nos encontramos a ambos realizando distintas transacciones en La Plata. Incluso se ve que ya hacían tratos juntos: el 28 de septiembre de 1598 Miguel de Burgos se obliga a pagar a su futuro compañero de compañía 180 pesos por una serie de géneros ${ }^{25}$. Para entonces, Miguel de Burgos ya era designado como comediante. Se ve que ambos se dirigen juntos hacia Lima, ya con la compañía en mente. De hecho, como se especifica en el documento del Callao, la escritura de concierto firmada con los músicos para la compañía, que al momento se halla en poder de uno de ellos, se hace nada más y nada menos que en Chuquiago (La Paz); es decir, con toda probabilidad, como será el modelo predominante en el siglo siguiente, los comediantes iban reclutando en el camino miembros para su tropa. En este caso, el camino iba a Lima desde Charcas. La compañía de Pérez de Robles y Miguel de Burgos, durante los siguientes años, retornará constantemente a Charcas; ya en 1601, de nuevo en La Plata, un niño huérfano de 14 años se asentó para trabajar como representante por un año con Pérez de Robles ${ }^{26}$.

Este tema daría para otro artículo. Ahora lo que me interesa es mostrar que hay base documental para sostener que los inicios de la profesionalización teatral en el Virreinato del Perú no tuvieron solamente a Lima como escenario. Es un fenómeno transterritorial complejo, en el cual Charcas participó activamente y desde muy temprano. Por eso, la documentación sobre Francisco de Morales es importante, porque él tenía un proyecto teatral en mente y el hecho de que haya en algún momento decidido mudarse a La Plata no es baladí.

Ya para cerrar, hago mención a un tema sobre el que todavía queda mucho trabajo por hacer y que es uno de los más relevantes para la reflexión que he emprendido. No se puede imaginar la estabilización del oficio teatral si no se piensa en lugares fijos de representación. En Lima, valga la redundancia, son los corrales que funda Morales los primeros que dan pie a la concretización de este proceso. Pero, ¿qué pasa en Charcas? ¿Tenemos que creer que los primeros corrales son el del Hospital de la Vera Cruz en Potosí (1616) y el del hospital de Santa Bárbara en La Plata (hacia finales de la década de 1620)? Marie Helmer (1960) dijo que quizá había ya uno en Potosí en 1572, pero esto es históricamente improbable, ella no documenta el dato y yo no he podido encontrarlo en los registros conservados para ese año.

Sin embargo, ¿cómo imaginarque, en Potosí, donde llegaban tantos autores de comedias, no había un espacio oficial para las representaciones hasta la segunda década del siglo XVII? Imposible. Más a la vista de lo que uno esperaría, se encuentra un dato que, además de abrir las puertas a futuras investigaciones, parece ofrecer una primera respuesta a esta cuestión. El 24 de septiembre de 1601 el virrey Luis de Velasco emitió la orden de que se conceda al Hospital de San Andrés de 
Lima el privilegio sobre las representaciones dramáticas. Para esto, manda que se construya ahí un corral. En el documento se detalla que Joan Gutiérrez de Molina, el administrador del hospital, al hacer petición del privilegio había explicado a su excelencia que: "todas las ciudades de España y México, y en este reino en la Villa Imperial de Potosí, los hospitales dellas tienen corrales y sitios donde se representan las comedias por el mucho aprovechamiento que dello se les resulta" 27 . Bastaría esa referencia para ilustrar el importante rol de la zona charqueña en la estabilización de lugares, modelos y circuitos de representación; en la estabilización de un oficio del cual, ya en la primera década del siglo XVII, un toque de talento de por medio, se podía vivir sin muchos apuros.

\section{Siglas de archivos citados}

ABAS: Archivo-Biblioteca Arquidiocesano Monseñor Miguel de los Santos Taborga.

ABNB-CPLA: Archivo y Biblioteca Nacional de Bolivia, Cabildo secular de Potosí.

ABNB-EP: Archivo y Biblioteca Nacional de Bolivia, Escribanías Públicas.

AGI: Archivo General de Indias

AGN: Archivo General de la Nación

APD: Archivo de la Provincia Dominicana de San Juan Bautista del Perú (Convento de Santo Domingo).

\section{Referencias citadas}

Barnadas, J. y A. Forenza (2000): “Noticias sobre el teatro en Charcas (siglos XVI-XIX)", Anuario del Archivo y Biblioteca Nacionales de Bolivia, pp. 557-575.

Betancourt, H. (1986): “El protocolo de Julián Bravo (1599): primer contrato de una agrupación teatral en América”. Latin America Theater Review 19, (2), pp. 17-22.

Bridikhina, E. (2007): Theatrum mundi. Entramados del poder en Charcas colonial, La Paz, IFEA / Plural.

Canet Vallés, J. L. (1997): “El nacimiento de una nueva profesión: los autores representantes (1540-1560)”, Edad de Oro, 16, pp. 109-120.

Durán Montero, M. A. (1990); “La entrada en Lima del virrey D. García Hurtado de Mendoza”, Laboratorio de Arte, 3, pp. 57-62.

Eichmann, A. e I. Arellano (2005): Entremeses loas y coloquios de Potosí (Colección del convento de Santa Teresa), Madrid / Frankfurt am Main, Iberoamericana / Vervuert.

Eichmann, A. (2006): "Notas sobre el teatro en Charcas", en Memoria del III Encuentro Internacional sobre Barroco, La Paz, pp. 333-345.

Eichmann A. (2008): "Nuevas notas sobre el teatro en Charcas”, Revista Ciencia y Cultura, 20, 2008.

Finque, S. (2019): “The Callao Contract of 1599: Actors Set the Stage for Early Modern Lima”, en Engel, E. A. ed., A Companion to Early Modern Lima, Brill's Compa- 
nions to the Americas, vol. 2. Leiden, Brill, pp. 424-441.

García García, B. (1996): “Las relaciones entre los comerciantesy artesanos del sector textil con la actividad teatral madrileña a fines del siglo XVI y principios delXVII", en de la Granja, A. y J. A. Martínez Berbel, eds., Mira de Amescua en candelero. Actas del congreso internacional sobre Mira de Amescua y el teatro español del siglo XVII, vol. 2, Granada, Universidad de Granada, pp. 159-170.

Gisbert, T. (1968): Esquema de la literatura virreinal en Bolivia, La Paz, Universidad Mayor de San Andrés, 1968.

Helmer, M. (1960): Apuntes sobre el teatro en la Villa Imperial de Potosí 1572- 1636, Cuaderno, 1, Potosí, Universidad Tomás Frías.

Lohmann Villena, G. (1945): El arte dramático en Lima, Madrid / Sevilla, Consejo Superior de Investigaciones científicas / Escuela de Estudios Hispanoamericanos.

Libros de Cabildos de Lima (1935-1968): 24 tomos, Bertram T Lee - Juan Bromley, eds., Lima, Consejo provincial de Lima.

Oleza, J. (1984): "Hipótesis sobre la génesis de la comedia barroca”, en Joan Oleza, ed., Teatro y prácticas escénicas, I, pp. 9-44.

Peña, B. (2016): “Fray Diego de Ocaña: Olvido, mentira y memoria”, Cuadernos de la América sin nombre, Alicante, Universidad de Alicante.

Quisbert, P. (2008): “Servir a Dios o vivir en el siglo: la vivencia de la religiosidad en ciudad de La Plata y la Villa Imperial (siglos XVI Y XVII)" en Eichmann, A. y
M. Inch, eds., La construcción de lo urbano en Potosí y la Plata (siglos XVIYXVII), Sucre, Ministerio de Cultura de España / ABNB, pp. 5-145.

Real Academia Española. Diccionario de Autoridades.

Rosenbach, A. S, W (1939): “The First Theatrical Company in America”. Antiquarian Society, 48, pp. 300-310.

Rostworowski, M. (2014): Historia del Tahuantinsuyo, Obras completas XVIII, Lima, Instituto de Estudios Peruanos.

Zugasti, M. (2005): La alegoría de América en el barroco hispánico: delarte efímero al teatro, Valencia, Pre-Textos.

Zugasti, M. (2008): “Teatro recuperado en Charcas: dos loas olvidadas de fray Juan de la Torre (OSA) a la entrada del virrey Diego Morcillo en Potosí”, en Arellano, I. y J. A. Rodríguez Garrido, eds., El teatro en la Hispanoamérica colonial, Madrid, Iberoamericana / Vervuert, pp. 295-321.

\section{Notas}

${ }^{1}$ Por el tipo de material que se conserva, la mayor parte de los estudios de este tipo se refieren a obras de finales del siglo XVII en adelante: en este contexto destacan, por ejemplo, las investigaciones de Miguel Zugasti (2005; 2008) sobre las piezas teatrales que se presentaron a inicios del siglo XVIII para la entrada del virrey Diego Morcillo a Potosí. Para lo que atañe al siglo XVII, quisiera resaltar, además de los trabajos referidos en el primer párrafo de este artículo, el estudio de Beatriz Peña (2016: 220-274) de la Comedia de Nuestra Señora de Guadalupe y sus milagros de fray Diego de Ocaña: obra representada por comediantes 
profesionales en 1601 en La Plata y Potosí en medio de una serie de festejos en honor a la instauración de la imagen de la virgen jerónima de Guadalupe en las susodichas ciudades.

2Por este motivo resultan importantes, para afrontar este tema, todos los estudios que tienen que ver con la financiación por parte de los Cabildos seculares de distintos tipos de espectáculos: como toda la serie de fiestas que tienen como objetivo la recreación de la presencia del monarca (recibimientos, juras, exequias, etc.) Para el caso charqueño destaca, sin duda, el trabajo de Eugenia Bridikhina (2007).

3Para la transcripción de documentos se modernizan todas aquellas grafías cuya modificación, por el estado de la lengua española en la época que nos atañe, no tenga trascendencia fonética.

4ABNB-CPLA, libro 6, fols. 166v-168v.

${ }^{5} \mathrm{ABNB}-\mathrm{CPLA}$, libro 5, fols. 84r-84v.

6Para la comprobación de este hecho bastaría remitirse al libro de Lohmann Villena (1945).

${ }^{7} \mathrm{AGN}$, PN, Juan Gutiérrez (1584), protocolo 75, fol. 681r-681v.

${ }^{8} \mathrm{LCL}$, Libro X, acta del 02/05/1586. He podido encontrar también noticias sobre algunos traslados suyos al Cuzco en 1587.

9AGI, LIMA, 108. El dato sobre la representación se encuentra en la serie de misivas que envía el Cabildo al Consejo de Indias para dar cuenta de los gastos que se hicieron en los recibimientos de virreyes como respuesta a una Cédula Real del 6 de junio de 1596. Sobre la suntuosidad del recibimiento aludido: India de virreyes y gobernadores del Perú, cap.16 (BNM, mss. 2835) o un resumen del acto central (Durán Montero, 1990).

${ }^{10} \mathrm{ABNB}$, EP. 41, fols. 245v- 246v.

${ }^{11 A B N B}$, EP. 70, fols. 582r-583v. El mismo día se firma también el traspaso del mesón y de la pulpería: ABNB, EP. 70, fols. 577r-581v.

${ }^{12} \mathrm{La}$ abasca es un tipo de tejido bastante popular que ya los incas, como explica María Rostworowski (2014), usaban para las prendas de la gente común. Estaría fabricado con lana de llama.

${ }^{13}$ Puede tratarse de alabardones para los caballos o, más probablemente, de "cualquiera cubierta que se pone encima de otra cosa, para preservarla y defenderla, como las cubiertas de encerado que se ponen encima de los tejadillos de los coches, y sobre las jaulas de los chucheros, con que salen a cazar al campo los pájaros" (Autoridades).

${ }^{14} \mathrm{ABNB}$, EP. 70, fols. 587r-587v.

${ }^{15} \mathrm{ABNB}$, EP. 70, fols. 608r-608v.

${ }^{16} \mathrm{ABNB}$, EP. 42, fols. 697v- 698r

${ }^{17} \mathrm{ABNB}$, EP. 70, fols. 748r- 749r; EP. 70, fols. 749v- 750r; EP. 42 , fols. $545 \mathrm{v}-546 \mathrm{r}$.

${ }^{18} \mathrm{APD}$, Libros de Autos, Libro XVI, Expediente 3. El arriendo del corral se hace a través de una enfiteusis por la cual se concede a Francisco de Morales y a su 
esposa el dominio útil del local 'por dos vidas'. Por su parte, el Cabildo secular, en su sesión del 14 de febrero de 1596, otorga a Morales licencia par realizar las construcciones en dicho espacio en la manera que solicitó (que no viene detallada) y aprobaron los comisarios y oficiales albañiles designados para tal efecto (LCL, Libro XII, acta del 14/02/1596).

${ }^{19} \mathrm{ABNB}$, EP. 60, fols. 221r- 222v.

${ }^{20} \mathrm{AGN}$, Cristóbal de Aguilar Mendieta (1595-1596), protocolo3, fols. $1244 \mathrm{v}-1245$.

${ }^{21}$ APD, Libros de Autos, Libro XVI, Expediente 3. Como dije precedentemente el arriendo firmado por Morales es una enfiteusis. La 'primera vida' que se concede al comediante y a su esposa debía contarse hasta la muerte del último de los dos. Lo que cede Morales a Casteloblanco (de quien poco sabemos) es el derecho a hacer uso de la siguiente vida. Sin embargo, Simón Fernández de Casteloblanco el 16 de octubre de 1599 declara que el corral pertence en realidad a Luis Fernández de Sosa; quien, a su vez, el 13 de enero de 1600 declara que pertenece a su suegra, María de Ribera, quien, en su testamento, lo deja en herencia a su hija, Ana de Ribera. La cual, sea dicho de paso, ya en el siglo XVII, es quien suele aparecer en la documentación teatral como la propietaria del corral.

${ }^{22} \mathrm{ABNB}$, EP, 83: fols. 367v - 369r. En marzo de este mismo año vende por 325 pesos ensayados, en la misma ciudad, un esclavo: ABNB, EP. 110: fols. 102r-104r.

${ }^{23}$ Lohmann Villena explica que el documento originalmente se encontraba en el archivo Nacional de Lima en los folios 250r-252v de la escribanía de Julián Bravoy que luego pasa a manos de D. B. T. Lee, quien se lo cede al coleccionista Rosenbach (1945: 78). El contrato está transcrito integramente por Helia Betancourt (1986).

${ }^{24}$ AGN, Antonio Corvalán (1597-1601), protocolo 25, fols. $265 \mathrm{r}-266 \mathrm{v}$.

${ }^{25}$ ABNB, EP. 82, fols. 429r-429v
${ }^{26}$ ABNB, EP. 84: fols. 593r - 594r.

${ }^{27}$ Documento transcrito por Lohmann Villena (1945: 91-93). El documento se encuentra en: AGI, Escribanía de Cámara, 503b, fols. 29v-32r. Forma parte de un expediente del cual se conservan 197 folios. Carátula del expediente: Lima año de 1613/ El hospital de señor/san Andrés de los reyes/ Legajo 8 de pleitos/ de Lima num. 9/ Alonso de Ávila y María del/ Castillo su mujer sobre el engaño de los/ asientos del corral de las comedias. 